\title{
Curso básico de didáctica de las ciencias enseñanza secundaria profesorado de ciencias en formación y en activo
}

\author{
Basic Course Teaching Science Secondary Education Science Teachers in Training \\ and Active
}

\section{Básico ciências curso de ensino ensino secundário professores de ciências em formação e active}

Adriana Patricia Gallego Torres ${ }^{1}$

\begin{abstract}
Resumen
Este escrito presenta una reseña del libro escrito por los investigadores Jaime Carrascosa Alís, Josep Lluís Domenech, Joaquín Martínez Torregrosa, Luis Osuna García y Rafaela Verdú Carbonell, y con ISBN: 978-84-616-9964-3.
\end{abstract}

Palabras Clave: Didáctica de las ciencias, desarrollo sostenible, formación de profesores.

\footnotetext{
Abstract

This paper presents a book review written by researchers: Jaime Carrascosa Alis, Josep Lluis Domenech, Joaquín Martínez Torregrosa, Luis Osuna
}

García and Verdú Carbonell and Rafaela ISBN: 978-84-616-9964-3.

Keywords: Teaching of science, sustainable development, teacher training.

\section{Resumo}

Este trabalho apresenta uma resenha do livro escrito por pesquisadores: Jaime Carrascosa Alis, Josep Lluis Domenech, Joaquín Martínez Torregrosa, Luis Osuna García e Verdú Carbonell e Rafaela ISBN: 978-84-616-9964-3.

Palavras chave: ensino da ciência, o desenvolvimento sustentável, formação de professores. 
¿Cómo implicar desde el primer momento a los profesores en formación en el estudio de los problemas que plantea la educación científica de los futuros ciudadanos y de sus posibles soluciones con el fin de mejorar el proceso de enseñanza y aprendizaje? Es la pregunta central que llevó a este grupo de investigadores en didáctica de las ciencias experimentales a escribir este libro, dirigido a seminarios de formación inicial o continua de docentes de ciencias.

El libro presenta los principales objetos de estudio que han hecho de la didáctica de las ciencias una disciplina científica. Los autores destacan que el trabajo que se presenta es parte del gran legado que dejó uno de los principales investigadores en didáctica de las ciencias experimentales en el mundo: Daniel Gil Pérez. En la década de 1980 instauró los principales problemas de la educación en ciencias, lo que más tarde Ilamaría educación científica de los ciudadanos, dada su gran preocupación por comprender los procesos de enseñabilidad, educabilidad y aprendizaje en los procesos formales desarrollados en las aulas de clase. Asimismo, nos mostró otra manera de ver la educación en ciencias hasta el punto de llegar a estudiar los procesos sociales de las ciencias y las tecnologías, lo que llamó un futuro sostenible y lo que poco a poco ha logrado el reconocimiento de un campo de conocimiento denominado ciencia de la sostenibilidad.

Los problemas a los que la educación científica hace referencia los autores los dividen en 12 capítulos, comenzando por el planteamiento de uno de los principales en la didáctica de las ciencias: ¿Qué hemos de saber y saber hacer los profesores de ciencias para desarrollar una enseñanza de calidad? Problema de investigación que ha sido planteado en diversos grupos de formación de Latinoamérica y el Caribe mediante la metodología denominada enseñanza aprendizaje por investigación. Dentro de las principales respuestas de los docentes, se hace referencia al desinterés de los estudiantes por la ciencia y su aprendizaje, la resolución de problemas, la evaluación, los contenidos curriculares, las prácticas de laboratorio, entre otras. Problemáticas que pueden ubicarse dentro de los diferentes campos de saber que componen la didáctica de las ciencias.

El segundo capítulo hace referencia a uno de los principales obstáculos para desarrollar procesos de enseñanza y aprendizaje de las ciencias. A este respecto, numerosas investigaciones en diferentes ámbitos han constatado la importancia de reflexionar en torno a las concepciones sobre la naturaleza de la ciencia y el trabajo científico no solo de los estudiantes sino también de los docentes, con el objeto de plantear diferentes procesos de innovación y desarrollo para poder conseguir una educación científica coherente con los desarrollos epistemológicos actuales.

El tercer capítulo hace referencia a la línea de investigación denominada prácticas de laboratorio. Todos somos conscientes de la necesidad de establecer un puente entre la teoría y la práctica, fundamentalmente por la gran capacidad que poseen para familiarizar a los estudiantes con la metodología científica. Sin embargo, las prácticas de laboratorio en lugar de ser un valor agregado en la enseñanza de las ciencias se han convertido en un foco de representaciones ligadas al método científico dibujando imágenes desvirtuadas de lo que es el trabajo científico y negando toda posibilidad de aprendizaje a los estudiantes.

El cuarto capítulo se encarga del problema de los problemas. Este quizás sea uno de los campos más desarrollados por la didáctica de las ciencias. Las investigaciones han llevado a los docentes al convencimiento de la importancia de plantear adecuadamente los problemas que le ponen a sus estudiantes, sus funciones, sus potencialidades $y$, sobre todo, la manera como orientan su resolución.

El capítulo quinto es uno de los problemas que más peso le dan los docentes cuando reflexionan en torno a su propia práctica y hace referencia al aprendizaje de los contenidos teóricos. Este capítulo inicia con la necesidad de reconocer la existencia de otros conocimientos elaborados previamente por los estudiantes antes de llegar a las 
aulas. Conocimientos que inicialmente se denominaron errores conceptuales y que hoy en día son considerados como otros tipos de conocimiento (ideas alternativas), los cuales deben tener un tratamiento adecuado. El capítulo presenta varias posibilidades para trabajar con los estudiantes en clase.

Los autores dedican el capítulo sexto a las relaciones ciencia, tecnología, sociedad y ambiente, destacando que en capítulos anteriores se han ocupado de aspectos claves en la enseñanza y aprendizaje. Sin embargo, existen otros aspectos que se hallan indisolublemente unidos a la naturaleza de la ciencia y el trabajo científico, como son las controvertidas relaciones entre la ciencia, la tecnología y la sociedad y sus implicaciones ambientales y planetarias. En este sentido, los autores plantean una serie de problemas concretos sobre los cuales se desarrolla el capítulo; estos son: ¿Qué interés tiene la inclusión en el currículo de las relaciones CTSA?, ¿qué visiones tienen los profesores de ciencias acerca de los problemas del mundo y de las decisiones a tomar?, ¿qué importancia tienen éstas visiones?, ¿a través de qué tipo de actividades y contenidos se puede favorecer una incorporación más efectiva de las relaciones CTSA? Los autores terminan el capítulo con varias actividades para la utilización de la historia de la ciencia.

El capítulo siete hace referencia a las actitudes hacia la ciencia y su aprendizaje. Esta problemática está relacionada con la negativa de los estudiantes por acercarse a la ciencia, a la metodología científica y su aprendizaje. Esto se ve reflejado no solo en las clases de ciencias, sino también se ha hecho evidente en los cursos de formación posgradual en diferentes disciplinas en las cuales se evidencia la actitud negativa de los estudiantes por enfrentarse a procesos de investigación. Lo que lleva a reforzar lo que muchos autores denunciaron en su momento: el interés de muchos estudiantes provenientes de una enseñanza tradicional se reduce significativamente con los años de escolaridad, hasta el punto de abandonar los estudios científicos. La gravedad del problema es tal, que aunque su afinidad no sean las ciencias, las consecuencias se reflejan en su vida profesional al no desarrollar una actitud positiva por el trabajo científico, esto unido a la pésima concepción que muchos elaboran de la ciencia y pésimos esquemas de resolución de problemas.

El capítulo ocho está dedicado a la evaluación, una problemática tan importante como controvertida. Los autores resaltan la importancia de este aspecto en el proceso de enseñanza aprendizaje tanto para los estudiantes como para los profesores. Cabe destacar que la didáctica de las ciencias ha realizado numerosas investigaciones en torno a esta problemática, usada muchas veces por los docentes como mecanismo para disciplinar a sus estudiantes y no como un instrumento de aprendizaje.

El capítulo noveno plantea la estructura problematizada de los temas y los cursos de ciencias. Los autores, para abordar este apartado, parten de la siguiente pregunta: ¿sobre qué problemas concretos ha de tratarse este tema? Al respecto plantean las siguientes preguntas complementarias: ¿cómo diseñar los contenidos de cada tema (o incluso de un curso completo), de forma coherente con el modelo aprendizaje como investigación orientada para favorecer el interés de los estudiantes hacia la ciencia, su participación en la construcción de los conocimientos y en definitiva, su inmersión en una cultura científica?; ¿Qué problemas de interés utilizar como problemas estructurantes para el desarrollo del curso y de cada tema?; ¿Qué secuencia de actividades concretas diseñar, considerando las metas a alcanzar y los obstáculos previsibles (teniendo en cuenta la investigación al respecto)? Estas preguntas tienen un origen común y la necesidad de problematizar los contenidos curriculares, para que realmente podamos trabajar en las clases de ciencias bajo la metodología de enseñanza aprendizaje por investigación. Los autores desarrollan de forma completa estas cuestiones y plantean como ejemplo de las mismas el capítulo diez, denominado "Luz y visión: ¿Cómo vemos? ¿Cómo podemos ver mejor?". 
El capítulo once está dedicado al complejo problema del cambio climático y la construcción de un futuro sostenible. Los autores advierten las graves consecuencias del cambio climático ya no solo para el planeta sino para la raza humana, que está en peligro de extinguirse. El problema es de tal magnitud que si no hacemos algo, no solo desde nuestra postura como docentes, sino como ciudadanos para poder construir un futuro sostenible, tendremos que seguir siendo víctimas de un sinfín de catástrofes naturales.

Para terminar, los autores plantean un apartado que, desde mi punto de vista, presenta una verdadera innovación en la didáctica de las ciencias. Es la necesidad de trabajar en la iniciación en la investigación y la innovación. En los cursos de formación inicial y continua hemos encontrado serios problemas a la hora de formular y fundamentar problemas de investigación, de realizar una adecuada construcción metodológica que conduzca a resolver el problema planteado, por nombrar solo algunas de los principales obstáculos que tienen los docentes a la hora de enfrentarse a la formulación y ejecución de proyectos de investigación. Este apartado representa un valioso aporte para quienes nos dedicamos a la formación de investigadores.

El libro es imprescindible para todo aquel interesado en la problemática de la enseñanza y aprendizaje de las ciencias tanto para la formación inicial de docentes, como la enseñanza continua y avanzada. Para obtener el libro o para comunicarse con los autores: Laime.carrascosa@uv.es 